\title{
On the Structure of the Helmholtz Layer and its Implications on Electrode Kinetics
}

\author{
H.J. Lewerenz \\ Joint Center for Artificial Photosynthesis, California Institute of Technology \\ Pasadena, CA 91125 USA
}

\begin{abstract}
Concepts and selected experiments on the structure of the Helmholtz double layer at the metal- and semiconductor - electrolyte phase boundary are reviewed. The widely used microcapacitor approach of the double layer and its limitations are assessed. Observations on the influence of the electrode potential on the energetic position of surface states at the Ag-electrolyte contact are compared to the predictions of classical charge transfer models that are based on transition state theory where adiabatic tunneling is assumed. Distance tunneling spectroscopy on $\mathrm{Au}(111)$ surfaces shows pronounced variations in tunneling barrier heights that are connected to the inner structure of the Helmholtz layer and implications on electrode kinetics are presented. At the semiconductor-electrolyte contact, the influence of the electrode potential on a charge injecting species that results in photocurrent doubling is reviewed for low- and higher doped $\mathrm{Si}(111)$ electrodes, showing that the complex that injects electrons into the conduction band is located outside the semiconductor surface. The observations are correlated with the search for low overpotential earth abundant electrocatalysts for solar fuel generation of solar fuels.

Key words: Helmholtz layer structure, electrochemical kinetics, electroreflectance, distance tunneling spectroscopy, electron injection, transition state concept
\end{abstract}

\section{Introduction}

The approximation of the Stern layer at the metal-electrolyte contact as a parallel plate capacitor with smeared out charges has a long history [1-3]. Early models on the charge transfer between a metal electrode and a redox electrolyte are still in use. They are based on the assumption of a homogeneous electrical field inside the double layer $[4,5]$ which only shows a dependency in the direction perpendicular to the electrode surface. Despite these relatively simplifying assumptions, the charge transfer at this phase boundary has been described quite convincingly by the Butler-Volmer equation and its simplified forms $[6,7]$. The parameter that describes electrode kinetics is represented by the pre-factor of this equation, i.e. the exchange current density. Presently, still the extrapolated exchange current density, $\mathrm{j}_{0}$, is used in the overall assessment of the kinetic properties of an electrocatalyst and, typically, so-called volcano plots are used to compare the various materials [8-10]. Since $\mathrm{j}_{0}$ denotes a current density, a determination of the actual electrode surface area is needed for an accurate comparison of the kinetic properties. Since the volcano plots use the metal-hydrogen or oxygen bond strength as the second parameter, present assessments of electrode kinetics are based on a simplified electrode-electrolyte model and on thermodynamic bonding calculations that do not take into account the solution phase. Although generally rather successful, these models have not resulted in a comprehensive understanding of the origin of the differences in catalytic activity for metals and intense efforts are needed to advance our present concepts, both, theoretically and experimentally. Such work can have tremendous impact on the development of solar fuel generators for carbon neutral renewable chemical energy $[11,12]$. 
In this communication, I will review some of the visionary experiments of Dieter M. Kolb and his group on well-defined metal single crystal-electrolyte interfaces which give new insights into the inner structure of Helmholtz double layers [13-17]. In addition, experiments by Jungblut et al., performed at the $\mathrm{Si}(111)$ - electrolyte junction, are presented that correlate well with the observations of Kolb et al. at $\operatorname{Ag}(100)$ - electrolyte junctions [18]. At the semiconductor interface, the influence of the electrode potential on a hitherto unidentified electron injecting complex is analyzed using differently doped samples which, in turn, results in different relative potential drop between semiconductor and the Helmholtz layer. For highly doped samples, the behavior approaches that of metals which establishes the connection of the Kolb and Jungblut experiments.

\section{Experimental}

All experiments have been performed on single crystal electrodes and the preparation of their surfaces has been described in the corresponding literature [13, 18]. Typically, the standard three electrode potentiostatic arrangement has been used for the work on $\mathrm{Ag}$ electrolyte and $\mathrm{Si}$ - electrolyte interfaces. The experimental arrangement for the electroreflectance and for the distance tunneling experiments will be shown in the respective section of this article.

Solutions were prepared from analytical grade or ultrapure chemicals and high purity water was used (typically 18M Ohm).

\section{On Classical Concepts in Electrode Kinetics}

\subsection{The metal-electrolyte interface}

Fig. 1 shows a visualization of a charged metal in contact with a redox electrolyte. The surface charge of the metal is confined to the Thomas Fermi screening length [19], which, for $\mathrm{Cu}$, is about 0.06nm [20], hence below an Angström for most metals. The ionic counter charge in solution consists of solvated ions of opposite charge as drawn in the figure. The extension of the interfacial double layer without specific adsorption is given by the plane through the counter ions and the metal surface. It is obvious that the counter ions are not charges that are smeared out and that a limiting area density of the solvated counter ions exists. This raises the question of the validity limits of the planar microcapacitor concept with smeared out charges on both sides.

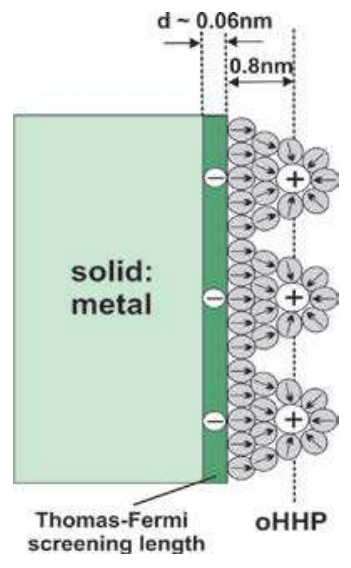

Fig.1: Schematic of the metal - redox electrolyte contact in the absence of specific adsorption (see text). 
We consider a mesh of ionic counter charge as shown in Fig.2. The charges $q$ are located as points at positions $d_{i}$ and separated equally in the plane through the center of the counter ions. Hence, the electric field between that plane and the electrode surface only shows a zdependence according to the geometry shown in Fig. 2.

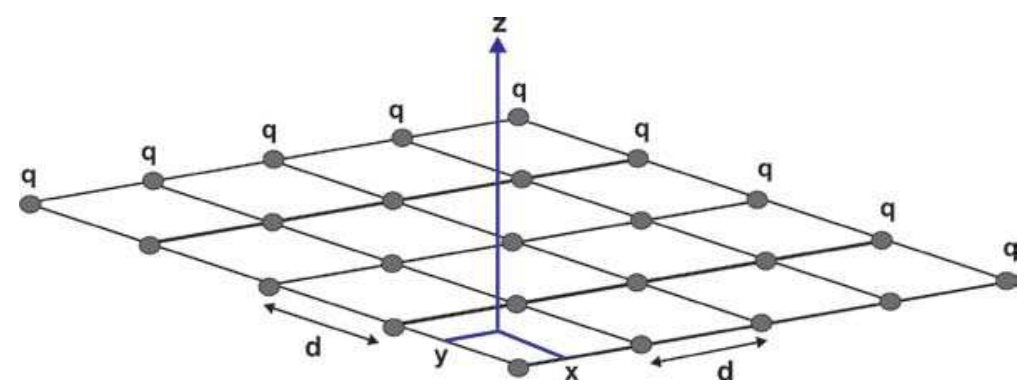

Fig.2: Mesh of point-like ionic counter charges q located in the xy plane in front of a metal electrode; distance of charges: $\mathrm{d}$; (see text).

For smeared out charges, the charge density is $\rho=\mathrm{q} / \mathrm{d}^{2}$ and the according electric field component in z-direction is $E_{z}=\rho / 2 \varepsilon \varepsilon_{0}$. For discrete charges, $E_{z}$ can be calculated using Poisson's summation formula [21, 22]:

$$
E_{z}(z)=\frac{\rho}{2 \varepsilon \varepsilon_{0}}\left[1+2\left(\cos \frac{2 \pi x}{d}+\cos \frac{2 \pi y}{d}\right) e^{-\frac{2 \pi z}{d}}+\ldots .\right]
$$

The electric field for discrete charges is modulated in $x-y$ direction as described by the cosine functions in eqn.(1) and has an exponential z-dependence, expressed by the decay length of the field $\lambda_{\mathrm{El}}=\mathrm{d} / 2 \pi$. Estimating the lateral distance of solvated ions as shown in Fig.1, yields a value of about $2 \mathrm{~nm}$, taking into account the extension of the solvation shell. Then, $\lambda_{\mathrm{El}} \sim 0.3 \mathrm{~nm}$. With the extension of the outer Helmholtz layer as indicated in Fig.1 $(0.6 \mathrm{~nm}-0.8 \mathrm{~nm})$, it already becomes clear that the influence of the lateral modulation at $\mathrm{z}=$ $\mathrm{d}_{\mathrm{HH}}$ (metal surface) is rather small, much less than $(1 / \mathrm{e}) E_{z}$. This effect becomes even smaller when the lateral distance between the counter ions decreases and at $\mathrm{z}=\mathrm{d} / 2(1 \mathrm{~nm}$ in the considered case), the difference to the smeared out case is only $17 \%$. Since, in reality, $\mathrm{z}<\mathrm{d} /$ $2(\sim 0.8 \mathrm{~nm})$, this difference is even smaller and for a dense layer of ionic counter charge, the smeared out model is a good approximation. On the metal side of the double layer, the assumption of a jellium-like behavior is often made [23]. This approximation holds for spmetals which can be theoretically described by a nearly-free electron approach assuming delocalized electronic charge and a smeared out background of positive charge from the nuclei (see Fig.3) 

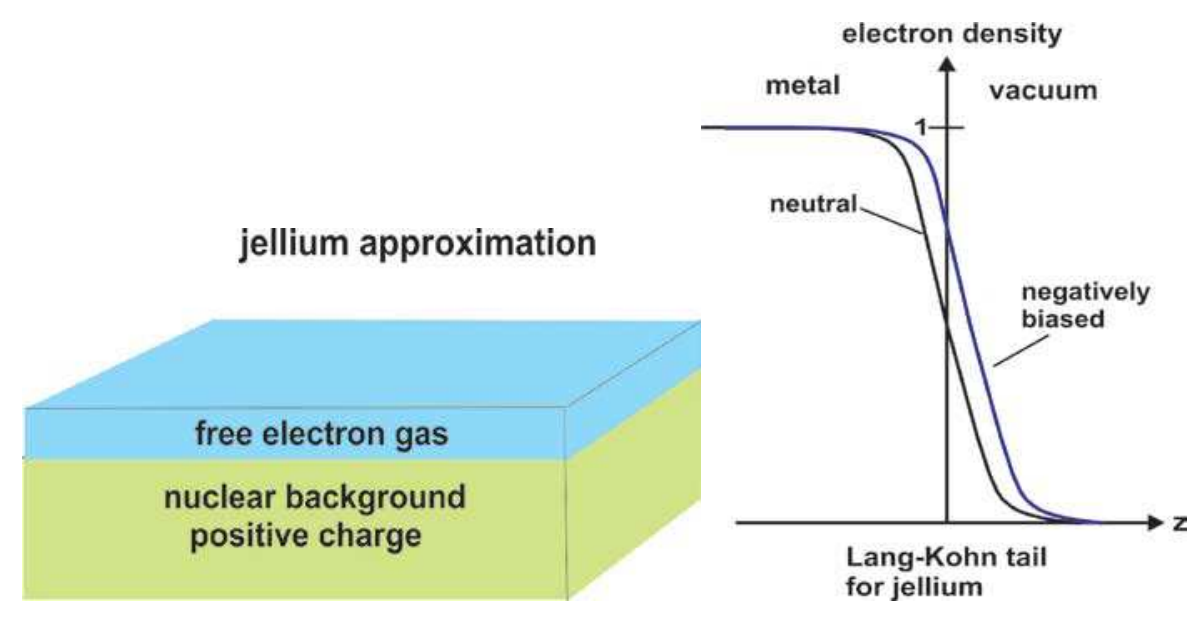

Fig.3: Schematic on the assumptions of the jellium approximation for simple metal electrodes (left), electron density perpendicular to the surface for the point of zero charge (pzc) and for negative bias (right).

As a result, the situation at the metal-electrolyte boundary can be visualized as shown in Fig.4, which are the basis of the historical concept of classical charge transfer models.
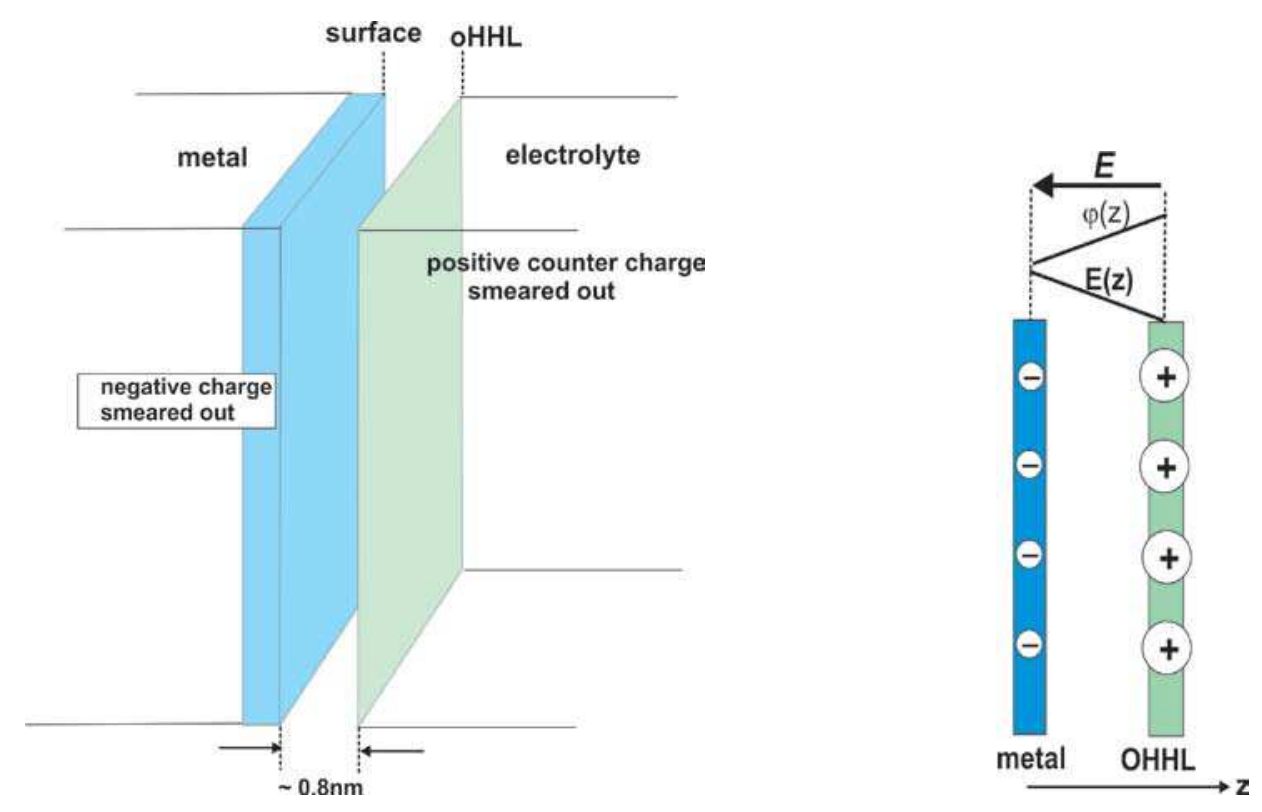

Fig.4: Metal-electrolyte double layer assumptions based on smeared out charges (left) and the resulting properties of the microcapacitor (right) with regard to the Galvani potential $\square$ (z), the electron energy $\mathrm{E}(\mathrm{z})$ and the constant electric field $E$.

\subsection{Note on activated complex theory}

Although clearly a textbook topic, the basic assumptions of transition state or activated complex theory are reviewed here, because, as will be shown below, the experiments of Kolb, and, separately of Jungblut, clearly demonstrate the electrochemical Stark effect that is at the basis of the assumptions of charge transfer theory. Considering a bimolecular reaction as shown in Fig.5, the existence of a transition state has been postulated rather early [24]. 


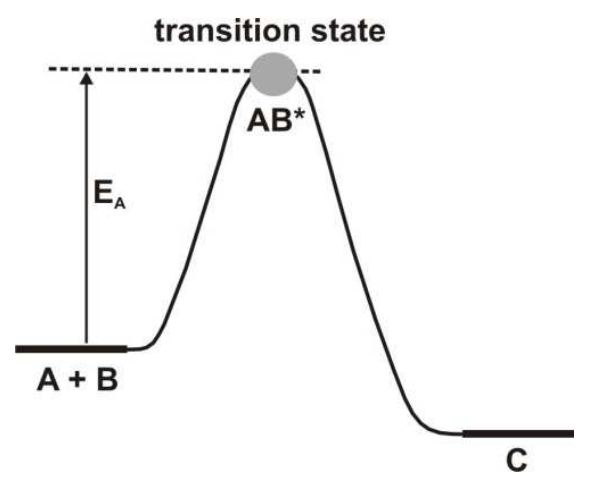

Fig.5: Model for a bimolecular reaction $A+B \underset{k_{r}}{\stackrel{k_{f}}{\leftrightarrow}} A B^{*} \rightarrow C \quad$ with a transition state $\mathrm{AB}^{*} ; \mathrm{k}_{\mathrm{f}}$ and $\mathrm{k}_{\mathrm{r}}$ denote the forward and reverse reaction rates (see text)

In electrochemistry, the influence of the electric field in the Helmholtz layer on the transition state energy has been used to describe electrode currents. The models are based on adiabatic electron tunneling where the tunneling probability is 1 , once the nuclear coordinates have reached their crossing position as shown in Fig.6. The change of the energetic position of the activated complex with electrode potential can then be determined by a triangulation as also shown in the figure.

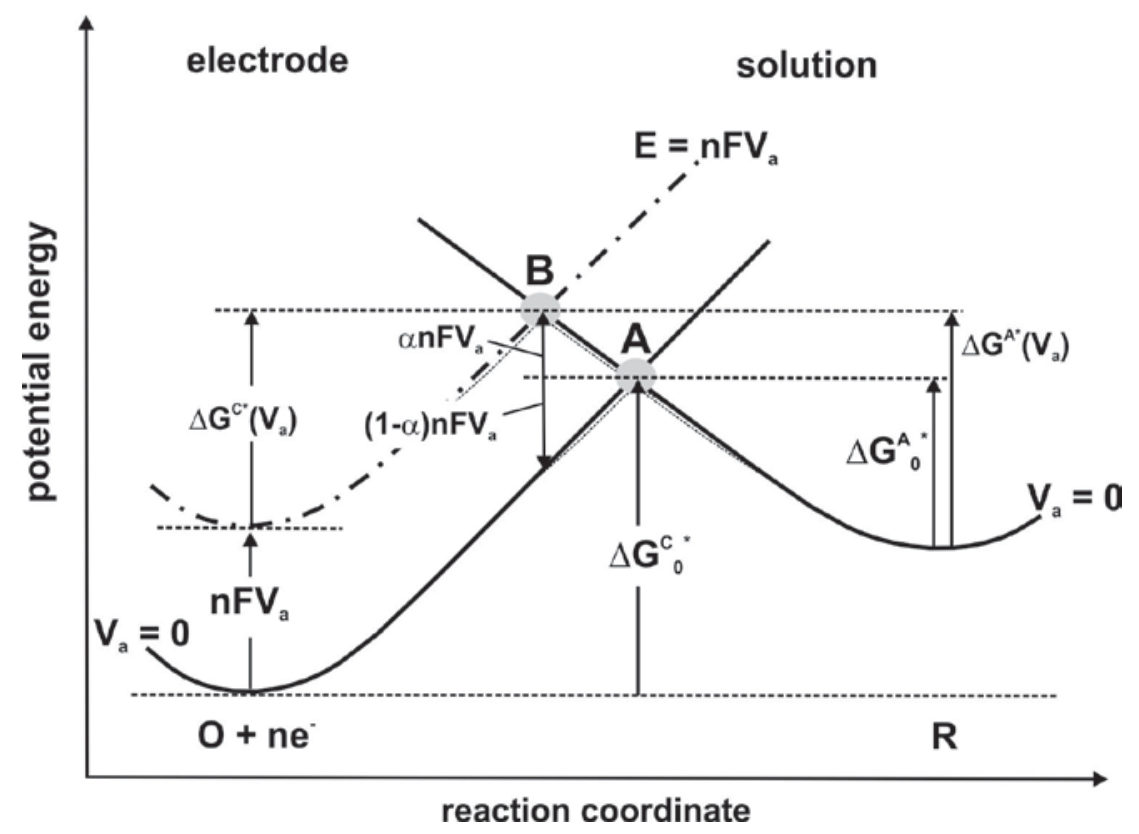

Fig.6: Influence of electrode potential at a metal on the energy of the transition state A and on the forward and reverse reaction energies; $\Delta \mathrm{G}^{\mathrm{C}, \mathrm{A}}$ : potential energies (Gibbs free energy) for the cathodic $(\mathrm{C}=$ and anodic $(\mathrm{A})$ reaction paths without applied potential; $\mathrm{V}_{\mathrm{A}}$ : applied potential; full lines: no applied potential; dashed-dotted line: with applied potential; $\alpha$ describes the partial influence of the potential on the energetic position of the transition state.

The influence of the potential on the transition state energy can be summarized by the expressions for the cathodic and the anodic Gibbs free energies:

$$
\begin{gathered}
\Delta G^{C^{*}}\left(V_{a}\right)=\Delta G_{0}^{C^{*}}-(1-\alpha) n F V_{a} \\
\Delta G^{A^{*}}\left(V_{a}\right)=\Delta G_{0}^{A^{*}}+\alpha n F V_{a}
\end{gathered}
$$


Insertion of eqns.(2,3) into the expressions for Arrhenius-type reaction rates and expression of electrochemical currents via these reaction rates results in the well-known Butler-Volmer equation that has found numerous applications in electrode kinetics [25, 26]. The assumptions made are, however, quite simplifying and, in addition, confirmation of electrochemical Stark effects has been scarce. In the following, in section 4, an experiment related to this topic, will be presented which in part can not be explained by the conventional theory.

\section{Electrochemical Stark Effect at $\operatorname{Ag}(100)$}

In this section, experiments that use metallic electroreflectance (ER) to identify the energetic position of surface states in a relative energy gap of Ag in the (100) direction of the surface Brillouin zone are presented [13]. The experimental arrangement and a few basic aspects of ER are outlined here first. Fig.7 shows the basic set-up and, in Fig.8, the influence of the potential modulation on the reflectance from a metal is schematically depicted.

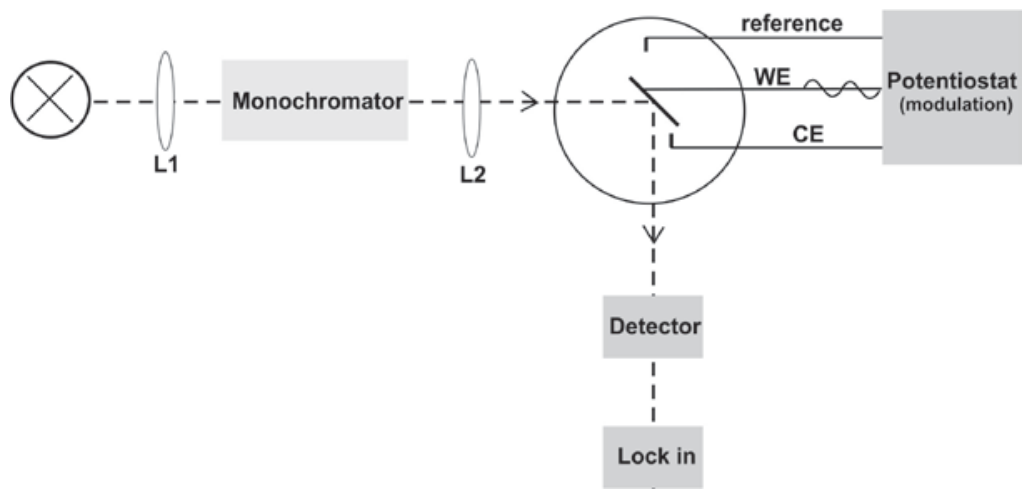

Fig.7: Typical experimental arrangement for potential modulated metallic electroreflectance; $\mathrm{L}_{1,2}$ : lenses; WE, $\mathrm{CE}$ : working- and counter electrode.
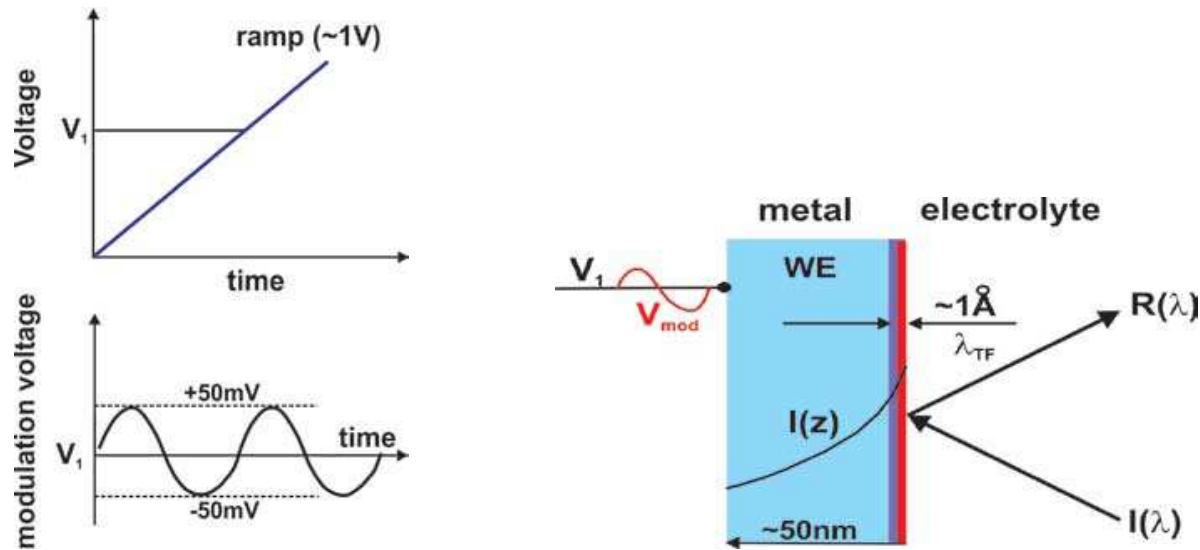

Fig.8: Potential modulation (left) and its influence on the reflectance $R(\lambda)$ at metal surfaces; $\mathrm{I}(\mathrm{z})$ : light intensity profile; $\mathrm{I}(\lambda)$ : incident light intensity at a given wavelength; $\lambda_{\mathrm{TF}}$ : Thomas Fermi screening length (see text).

Although the impinging spectral light penetrates typically several ten nanometers into the metal, depending on the optical properties, such as Drude absorption [27] and interband transitions, the potential modulation is spatially restricted to the topmost surface due to the extremely small Thomas Fermi screening length for static electric fields and, with a modulation frequency in the range of a few $10 \mathrm{~Hz}$, these fields are static with regard to the ultrafast electron gas response. 
Fig.9 shows the influence of the modulation with regard to the Lang Kohn electron density tail: the electron density at the surface is modulated around the value that is given by the fixed applied potential $\mathrm{V}_{1}$ (compare Fig.8).

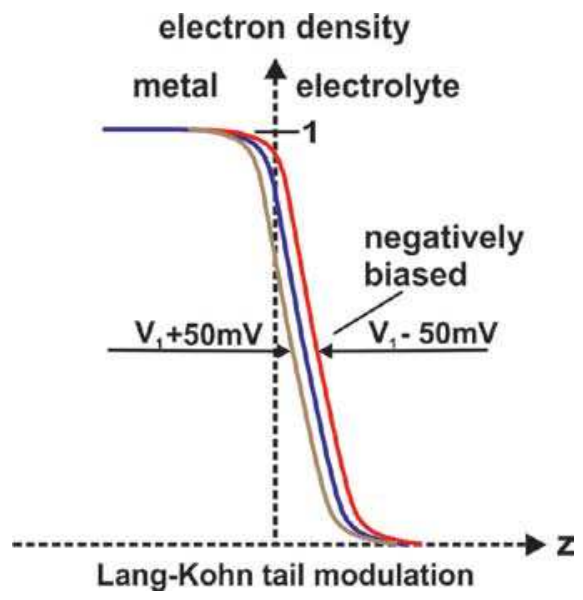

Fig.9: Visualization of the change of the surface electron density by potential modulation at an applied cathodic bias $\mathrm{V}_{1}$; the vertical dotted line denotes the geometric surface position without any bias; black line: electron density profile for $\mathrm{V}=\mathrm{V}_{1}$; red lines: modulation induced electron density shifts (see text).

Due to this modulation, the translational invariance in z-direction is lost and vertical (kconserving) transitions within the reduced zone scheme spread over a range of initial and final state wave functions which smears out the structure in the reflectance spectrum and the difference spectrum shows changes in sign (see for example, refs. [28-32]). In Fig.10, this effect is indicated and a electroreflectance spectrum is shown.

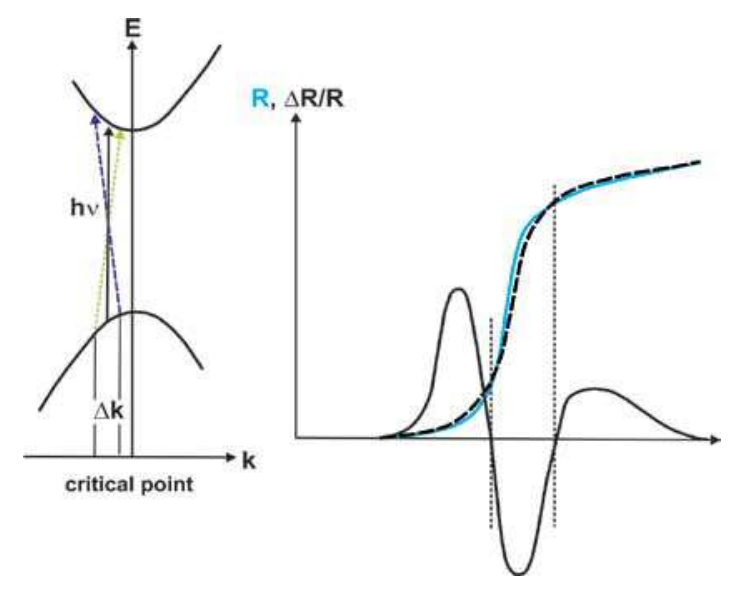

Fig.10: Origin of ER spectra; left: schematic of the relaxation of k-conservation by $\Delta \mathrm{k}$ near a critical point in the $\mathrm{E}(\mathrm{k})$ band structure due to loss of translational symmetry in z-direction by potential modulation; right: resulting influence on the reflectance spectrum $\mathrm{R}$ (dashed line, modulated by the blue line) and resulting ER spectrum with sign inversion.

The ER spectra are described by the change of the optical constants $\varepsilon_{1}$ and $\varepsilon_{2}$ and, also by parameters $\alpha$ and $\beta$ that contain functions of the optical constants:

$$
\frac{\Delta R}{R}=\frac{1}{R} \frac{d R}{d V_{\mathrm{mod}}}=\alpha \Delta \varepsilon_{1}+\beta \Delta \varepsilon_{2}
$$


It can be shown that the inflection of the ER signal near a parabolic edge reflects rather well the corresponding transition energy $[13,14]$. The data that will be presented below have been obtained by this assignment.

We now consider the k-space energy band structure of Ag and focus on the surface Brillouin zone, constructed from the fcc bulk Brillouin zone as shown in Fig.11.

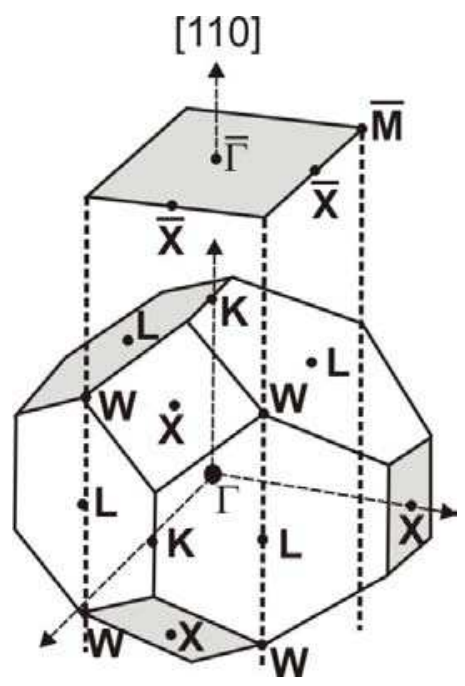

Fig.11: Construction of the fcc-related surface Brillouin zone in [110] direction; X, L, K, W are symmetry points of the bulk Brillouin zone; X denotes (100), L (111), K (110) symmetries; $\Gamma$ denotes the center of the bulk Brillouin zone; the surface Brillouin zone symmetry points are labeled by a bar on top of the symbol.

The k-space region of the band structure of interest for the ER experiments is located between $\bar{\Gamma}, \bar{X}, \bar{M}$. Fig. 12 shows the according section of the band structure and, also, the surface states, labeled A and B that occur in the relative energy gap near the [100] direction.

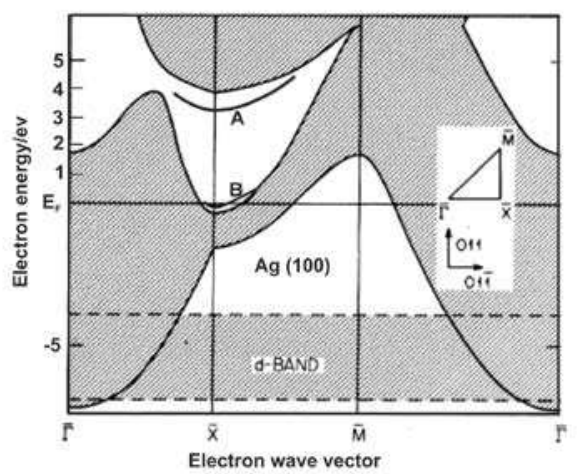

Fig.12: Energy bands at the surface Brillouin zone of Ag in the section displayed in the insert (from ref. [13]), showing the surface states (bands) A and B. Note that the relative energy gap near (100) is of the order of $4 \mathrm{eV}$.

The ER experiment was done by polarizing the Ag electrode mostly positive from pzc and measuring the ER signal related to the states A and B. It is found that the electrode potential exerts a strong influence on the energy of these states (it is known that bulk Bloch-like states are much less susceptible to surface potential changes, due to their extension into the bulk, and, actually, ER experiments also reveal bulk band structure features despite the ultrahigh surface sensitivity [33, 34].). In Fig.13, the results are summarized. 


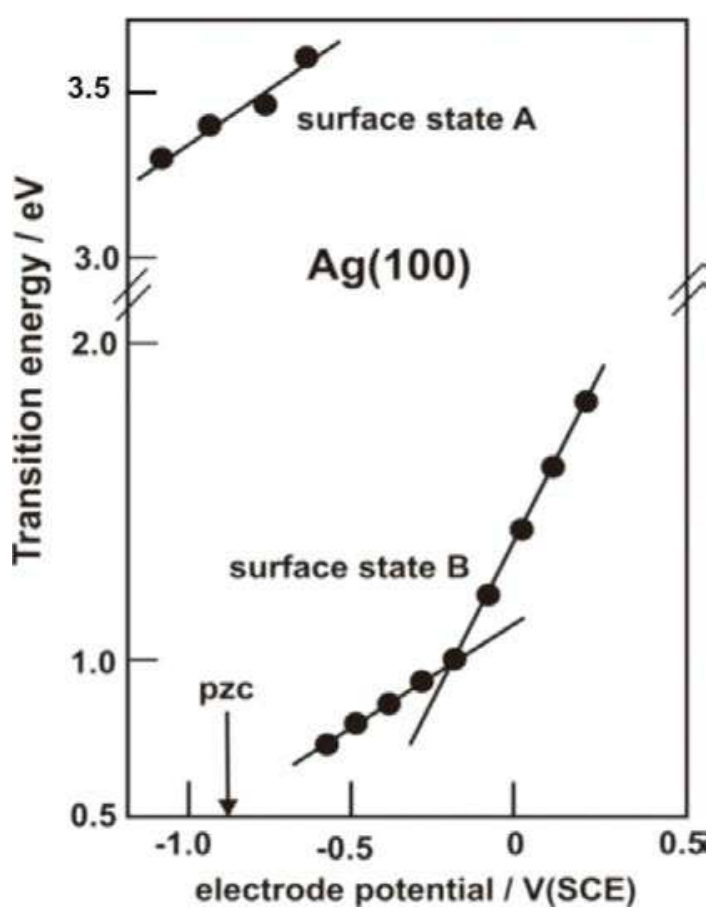

Fig.13: Evaluation of electroreflectance measurements on the change of the energy of the surface states A and B of Ag (see Fig.12) induced by electrode polarization; note that a slope of $<1$ is observed at potentials closer to pzc whereas stat B shows an increase beyond that value for more anodic potentials.

With applied anodic potential, the transition energies from occupied states into the surface states increases. One observes a slope of 0.7 for the surface state A and a similar slope for state $\mathrm{B}$ for potentials below $0.8 \mathrm{~V}$ (SCE). At more anodic potential, the slope becomes larger 1, reaching a value of close to 2 . The applied voltage results in an altered potential drop at the $\mathrm{Ag}$ - electrolyte interface. The surface states, however, is different and at least for the lower anodic potentials, the behavior can be explained by the linear models presented above and in Figs.4 and 6. One observes a relative change of the surface state energy with regard to that of the bulk band structure as indicated in Fig.14. The red arrows indicate the transition energies near the pzc whereas the blue arrows symbolize the transitions for applied potential of $0.8 \mathrm{~V}$. The Fermi level for the applied potential is given on the right hand side of the figure. The optical transitions now occur into the states $\mathrm{A}^{*}$ and $\mathrm{B}^{*}$ as shown in Fig.14. For lower anodic potentials, these results demonstrate that the electric field in the Helmholtz layer influences the energetic of eigenstates that extend or reside in the double layer. The shift of about $0.7 \mathrm{eV}$ of the transition energies with $1 \mathrm{~V}$ applied potential can be described within the approximations made for the derivation of the Butler-Volmer equation, assuming an asymmetry coefficient of 0.7 . This indicates that the states protrude to some extend into the Helmholtz double layer but are located closer to the electrode than towards the outer Helmholtz layer as would be expected from their nature as surface states. Fig.15 shows a schematic of this effect.

The superlinear behavior of state B to $\mathrm{B}^{*}$ can not be explained within the microcapacitor approximation. D.M. Kolb himself explained the observation, made in the early 1980s, by stating: "a shift greater than unity can only be understood in terms of the discrete nature of the water dipoles and specifically adsorbed ions which has been shown to cause oscillations and overshooting of the potential next to a metal surface" [13]. We will see below that this 
discrete nature of the double layer has indeed an influence on the potential distribution which has been examined more than two decades later.

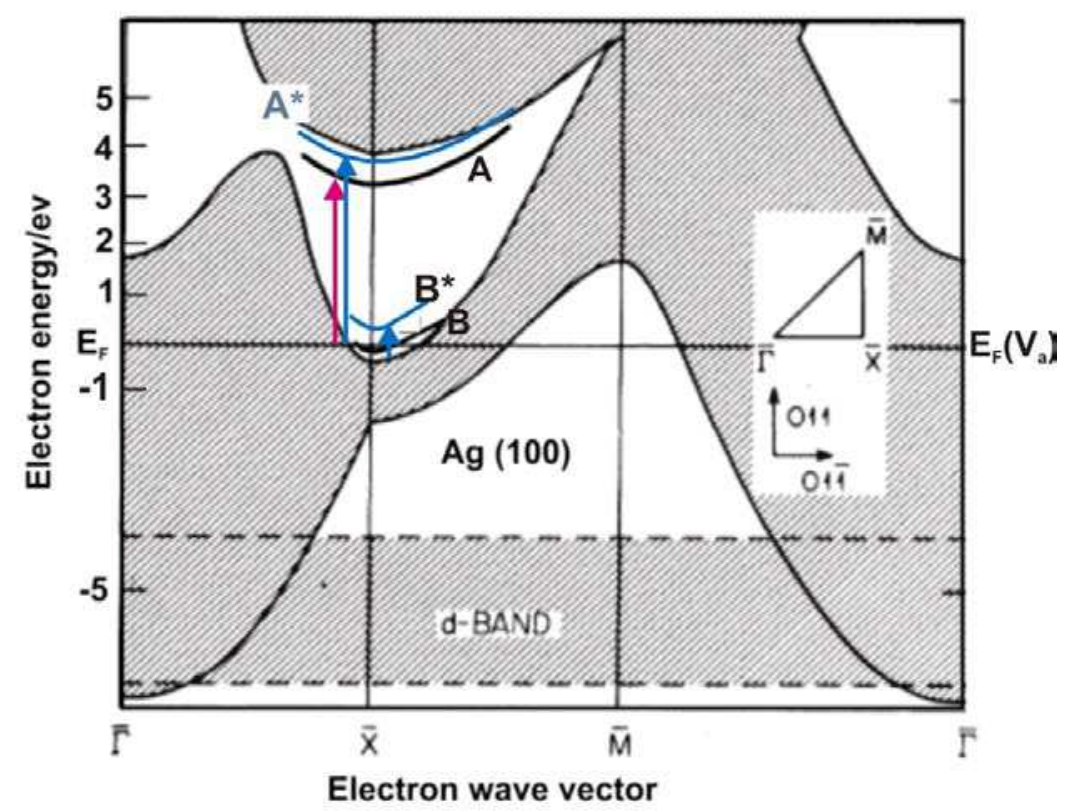

Fig.14: Energy scheme on the influence of the applied potential on the transition energies between occupied bulk states and the surface states A, B, measure by ER; red arrows: situation before applying anodic bias; blue arrows: after biasing to about $+0.8 \mathrm{~V}$ vs. pzc; note the relative shift of the surface states with regard to the bulk bands and the re-labeling of the states as $\mathrm{A}^{*}$ and $\mathrm{B}^{*}($ see text).

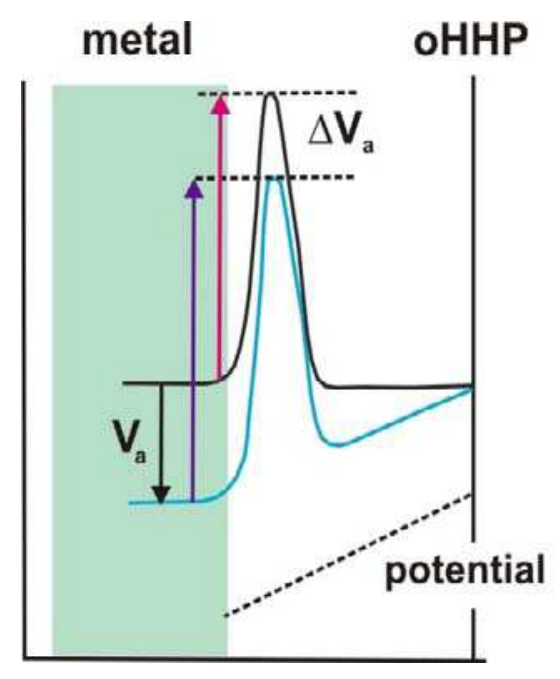

Fig.15: Energy schematic on the influence of the applied potential on the bulk Ag states and on the surface states; whereas the bulk bands shift with potential due to the Helmholtz layer potential drop, the surface states show a reduced change, labeled $\Delta \mathrm{V}_{\mathrm{a}}$, resulting in an increased energetic difference between the bulk initial states for the ER transition and the surface states $A^{*}, B^{*}$.

\section{Doping dependence of electron injection into $\mathrm{Si}(111)$}

At the semiconductor-electrolyte interface, the potential and energy relations differ principally from that at the metal contact. For semiconductors, doped in the range between 
$10^{14}-0^{17} \mathrm{~cm}^{-3}$, the capacitance of the space charge layer is much smaller than that of the Helmholtz layer and the contact potential drop across such junctions is expressed by

$$
V_{C}=\frac{Q}{C}=Q\left(\frac{1}{C_{H H}}+\frac{1}{C_{S C}}\right)
$$

where $\mathrm{Q}$ denotes the total charge, $\mathrm{C}$ the capacitance, $\mathrm{C}_{\mathrm{HH}}$ and $\mathrm{C}_{\mathrm{SC}}$ are the Helmholtz- and space charge layer capacitance, respectively. The former has, in the case of semiconductors, values of $C_{H H} \sim 0.5-3 \mu \mathrm{Fcm}^{-2}$ whereas the space charge layer capacitance, depending on the doping level is $C_{S C} \approx 1-10 \mathrm{Fcm}^{-2}$. In eqn.(5), surface states have not been considered. They can alter the energy relations considerably. Since here, the behavior of hydrogen terminated surfaces upon anodization is analyzed, the contribution of surface states has been neglected although this is an approximation that only holds for potentials slightly anodic of open circuit in dilute ammonium fluoride solutions. For low enough doped semiconductors, the potential drop in the Helmholtz layer is about 0.01 of that in the semiconductor surface as is shown in Fig.16.

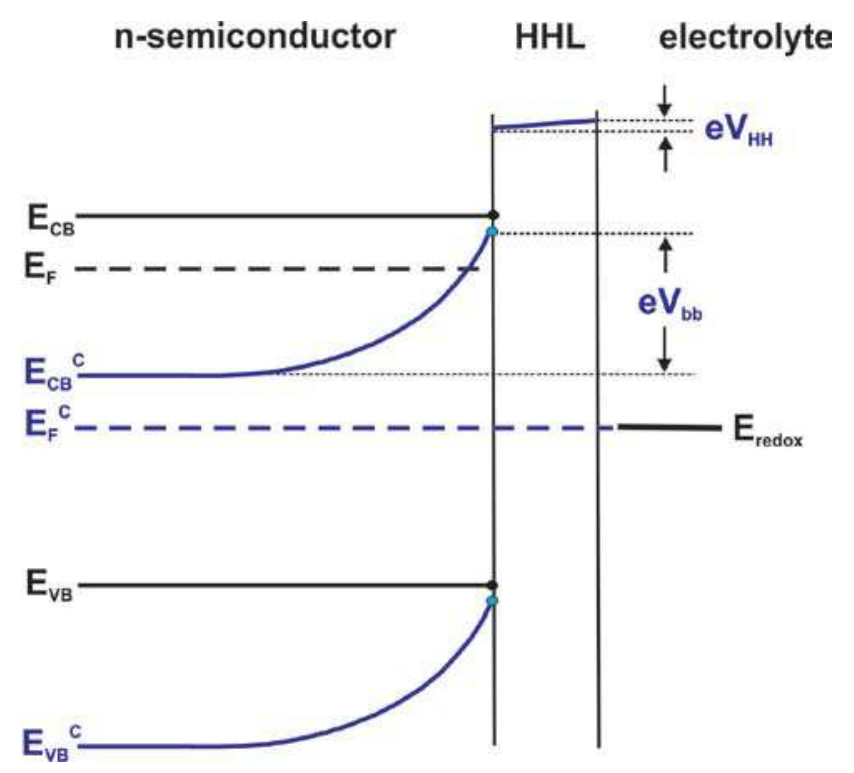

Fig.16: Energy relation with respect to the contact potential difference given by $E_{F}-E_{r e d o x}$, at the semiconductor-electrolyte contact; $\mathrm{E}_{\mathrm{CB}}, \mathrm{E}_{\mathrm{VB}}$, conduction- and valence band edges, respectively; superscript $\mathrm{c}$ indicates the energy band positions after contact formation; $\mathrm{eV}_{\mathrm{HH}}$, $\mathrm{eV}_{\mathrm{bb}}$ denote the energy drop across the Helmholtz layer and the semiconductor space charge (band bending), respectively.

Below, the dark current-voltage characteristics for differently doped n-Si are analyzed. The results are shown in Fig.17. These curves show a surprising resemblance of the photocurrent-voltage characteristic of $\mathrm{n}-\mathrm{Si}$ in dilute $\mathrm{NH}_{4} \mathrm{~F}$ solutions. Generally the photocurrent behavior is characterized by three potential areas that relate to different surface transformation phenomena [35]: in the region slightly anodic from ocp, before the first current maximum, so-called divalent dissolution prevails. It could be shown that only two charges are needed to dissolve $\mathrm{Si}$ and, in this potential regime, porous $\mathrm{Si}$ formation occurs [36]. The according reaction is

$$
S i_{S}+h_{V B}^{+}(h v)+6 H F \rightarrow S i F_{6}^{2-}+H_{2}+4 H_{a q}^{+}+e_{C B}^{-}
$$


where a light-generated hole from the valence band and an injected electron from solution into the conduction band contribute to the dissolution process. Accordingly, photocurrent doubling occurs in this potential region since for each generated hole, two charges contribute to the process. Also, hydrogen evolution is observed at potentials anodic from ocp (see eqn.(6). Surface analyses with photoelectron spectroscopy have shown that the surface is free of oxidized $\mathrm{Si}$, except for $\mathrm{OH}$ groups that partly terminate kink site atoms [37, 38]. Near the first photocurrent maximum, tetravalent dissolution sets in according to

$$
\mathrm{Si}+2 \mathrm{H}_{2} \mathrm{O}+4 h_{V B}^{+}(h v) \rightarrow \mathrm{SiO}_{2}+4 \mathrm{H}_{a q}^{+}
$$

where four light-generated holes oxidize $\mathrm{Si}$ in the presence of water. In the region of the second current maximum electropolishing occurs and a thin oxide film is residing on the continuously dissolving Si surface. In this case the oxide etch rate and the Si oxidation rate equilibrate and, due to 3-dimensional etching of roughened areas, smoothing of the surface results. In the third regime, at higher anodic potentials, photocurrent oscillations are observed [39-41]. They are a result of a non local oxidation and etch rate distribution that leads to pore formation in the oxide that covers the Si surface at these potentials. Conclusive theoretical models have been developed to describe this phenomenon. Fig.17 shows a set of dark current-voltage characteristics for higher doped n-Si samples as indicated in the figure.

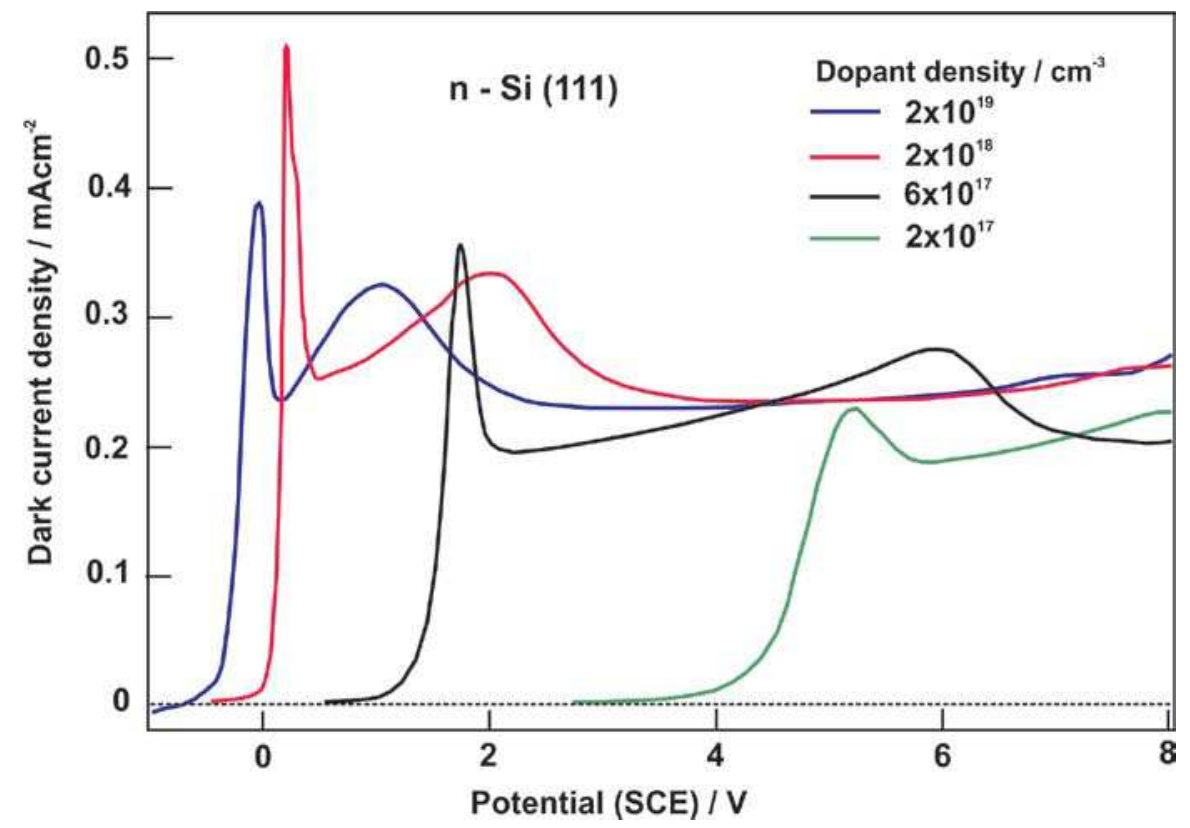

Fig.17: Dark current-voltage curves for differently doped n-Si(111) samples in $0.1 \mathrm{M} \mathrm{NH}_{4} \mathrm{~F}$ solution at $\mathrm{pH} 4$ (see text).

One observes that with increasing doping level, the onset of the current moves to lower anodic potentials and, for the highest doping level of $2 \times 10^{19} \mathrm{~cm}^{-3}$, the characteristic looks virtually identical to that of a low doped $\left(10^{14}-10^{15} \mathrm{~cm}^{-3}\right.$ range) illuminated sample. Taking the mass action law of semiconductor physics, i.e. $n \cdot p=n_{i}^{2}\left(n_{i}\right.$ intrinsic carrier concentration), there are too little holes available to sustain the currents of several hundred microampere per $\mathrm{cm}^{2}$. Indeed, for lower doped samples, the electrodes show excellent blocking behavior for this reverse bias direction of an n-type semiconductor. Accordingly, the currents seen in Fig. 17 must result from the electron injection process mentioned above that, on illuminated n- 
Si, leads to photocurrent doubling. We now consider the energy relations for the various doped samples. Due to the difference in doping level, the space charge capacitance of each sample is different and, consequently (compare eqn.(5) the potential drop in the Helmholtz layer will change. As experimental data, we have the shift of the onset of the current with doping, $\mathrm{V}_{\mathrm{on}}-\mathrm{V}_{\mathrm{fb}}$ with regard to the flatband potential. The latter is known for $\mathrm{H}$-terminated $\mathrm{Si}$ and the values for higher doped samples have been calculated taking into account the deviation from the Boltzmann approximation for very high doped samples (using the FermiDirac integral values). For the determination of the respective potential drop in the space charge layer, $\mathrm{V}_{\mathrm{SC}}$, we use the expression [42]:

$$
C_{H H}\left(\begin{array}{ll}
V & V_{f b}
\end{array}\right) \quad V_{S C} \quad \sqrt{2 N_{D} S i \quad{ }_{0} k T} e^{\frac{q V_{S C}}{k T}} \frac{q V_{S C}}{k T} 1^{1 / 2}
$$

and Table I shows the evaluation.

\begin{tabular}{rccccccc}
\hline $10 \cdot{ }^{17} N_{\mathrm{D}} / \mathrm{cm}^{-3}$ & $\mathrm{~V}_{\mathrm{on}} / \mathrm{V}$ & $\mathrm{V}_{\mathrm{fb}} / \mathrm{V}$ & $\mathrm{V}_{\mathrm{ont}}-\mathrm{V}_{\mathrm{ft}} /$ & $\Delta \mathrm{V}_{\mathrm{sc}} / \mathrm{V}$ & $\Delta \mathrm{V}_{\mathrm{HH}} / \mathrm{V}$ & $10^{6} \mathrm{~W} / \mathrm{cm}$ & $10^{7} E_{\max } / \mathrm{V} \mathrm{cm}^{-1}$ \\
\hline 2.0 & 4.37 & -0.73 & 5.10 & 4.56 & 0.54 & 17.0 & 5.2 \\
6.0 & 1.46 & -0.76 & 2.22 & 1.66 & 0.56 & 6.0 & 5.6 \\
20.0 & 0.10 & -0.80 & 0.90 & 0.38 & 0.52 & 1.6 & 4.7 \\
200.0 & -0.34 & -0.86 & 0.52 & 0.05 & 0.47 & 0.18 & 5.4 \\
\hline
\end{tabular}

Table I: Determination of the relative potential drop in the space charge layer of differently doped $n$-Si samples and the according potential drop $\mathrm{V}_{\mathrm{HH}}$ in the Helmholtz double layer (see text).

The left four columns give doping level, onset potential for the current, the flatband potential and the difference $V_{\text {on }}-V_{\text {fb }}$. The fifth column shows the calculated potential drop in the space charge region that varies drastically with doping level. Using the expression

$$
V_{H H} \quad\left(\begin{array}{lll}
V_{o n} & \left.V_{f b}\right) & V_{S C}
\end{array}\right.
$$

the sixth column shows that the potential drop in the Helmholtz layer, $\mathrm{V}_{\mathrm{HH}}$, is almost identical for all doping levels. The corresponding electric field (right column) is of the order of $5 \times 10^{7}$ $\mathrm{Vcm}^{-1}$. Fig. 18 summarizes the findings.

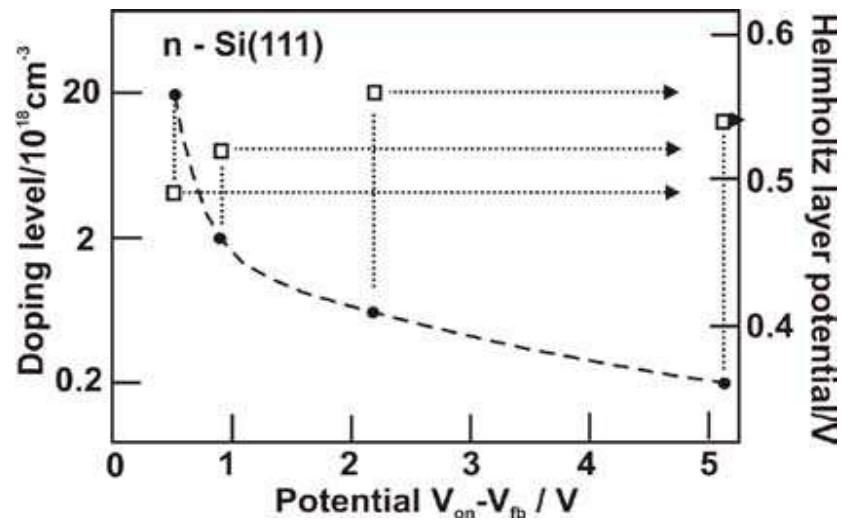

Fig.18: Plot of doping level versus dark current onset potential and the according calculated potential drop in the Helmholtz layer (see text). 
The result indicates that a certain electric field is necessary for the electron injecting complex to be activated. In former experiments using intensity modulated photocurrent spectroscopy [43], a position of about $0.2 \mathrm{eV}-0.3 \mathrm{eV}$ below the conduction band edge was assumed. This is in accordance with the observations made here and Fig.19 shows an energy scheme for the switching-on of the electron injection process. The scheme also shows that the injecting complex is likely not bound to the electrode but rather residing within the double layer because otherwise, the differences in Helmholtz layer field strength acting on the semiconductor surface and on the complex would not be pronounced enough. For high doping, the band edges of the semiconductor shift downwards due to the electric field in the Helmholtz layer. The according shift for the complex is smaller, eventually allowing electron injection if the energy of the electron donor complex is located above the band edges. This experiment is a further demonstration of electrochemical Stark effects and it can be explained based on the classical microcapaitor model. It should be noted that the surface chemistry, induced by electron injection is hitherto unknown, except for the divalent region where pore formation has been observed [18]. Therefore, high surface resolution synchrotron radiation photoelectron spectroscopy experiments are planned.

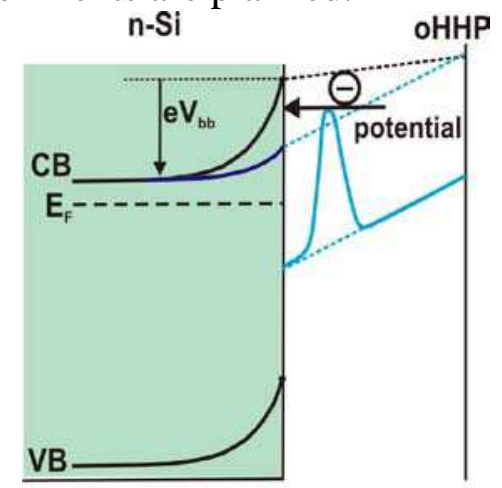

Fig.19: Energy relations for the activation of an electron injecting complex residing in front of n-Si; black lines: situation for low doped semiconductor (compare Fig.16); blue lines: situation for high doping with a corresponding shift of the band edges that results in an energetically favorable position of the electron injecting species which is less affected by the applied field.

In the next section, results are presented that show that the inner structure of electrolyte double layers has also to be considered for charge transfer processes.

\section{Distance Tunneling Spectroscopy}

Among the variations of scanning tunneling microscopy methods, distance tunneling spectroscopy (DTS) allows the analysis of tunneling barrier heights. The experimental arrangement of the method is shown in Fig.20.

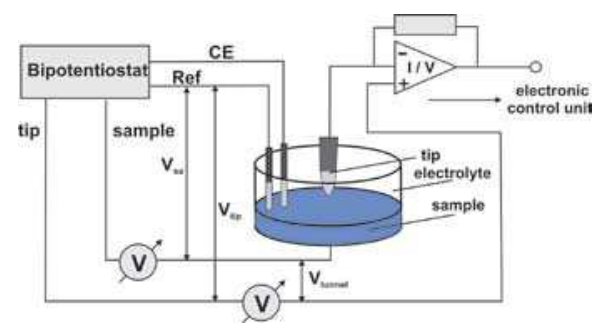

Fig.20: Experimental set-up for scanning and distance tunneling spectroscopy measurements after ref. [44]. The bipotentiostat allows control of the sample potential and application of the 
potential to the tip for tunneling as well as measurement of the tunneling current shown in the upper right of the figure.

The experiment was performed on well-defined $\mathrm{Au}(111)$ surfaces in $0.1 \mathrm{M} \mathrm{H}_{2} \mathrm{SO}_{4}$ for three applied potentials: at open circuit, and negative and positive from ocp. The tip was brought in close contact to the sample and then withdrawn without altering the position. The distance dependent tunneling current is given by

$$
I_{T}=\frac{1}{R_{0}} V_{T} e^{-A \sqrt{\phi_{T}} z}
$$

and the tunneling barrier height, $\Phi_{\mathrm{T}}$, is then obtained from the distance dependence of $\mathrm{I}_{\mathrm{T}}$ :

$$
\Phi_{T}(z)=\frac{\hbar^{2}}{8 m}\left(\frac{d \ln I_{T}}{d z}\right)^{2}
$$

From the measurement, shown in Fig.21, the tunneling barrier heights have been determined as seen in Fig.22. Potentials have been varied only in the double layer regime where no electrochemical reactions occur.

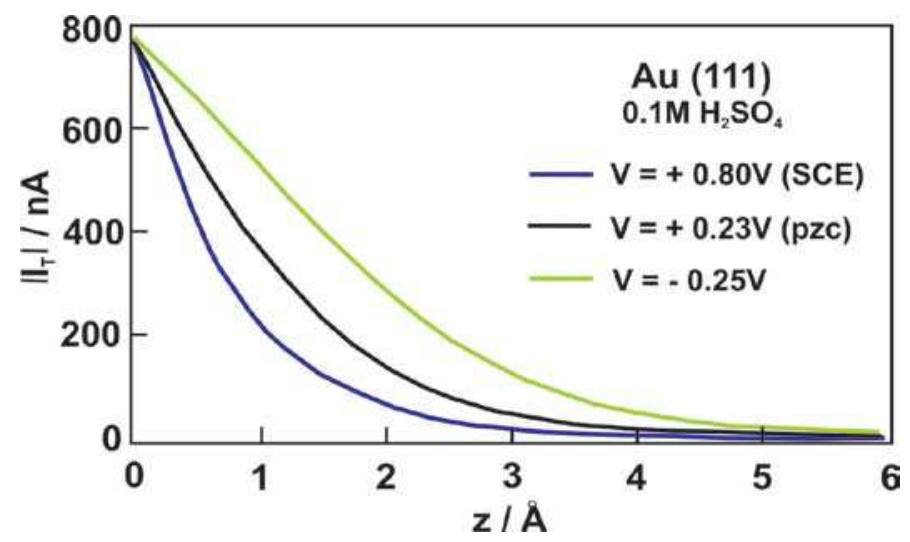

Fig.21: Distance tunneling currents for three electrode potentials as indicated in the figure (see text).

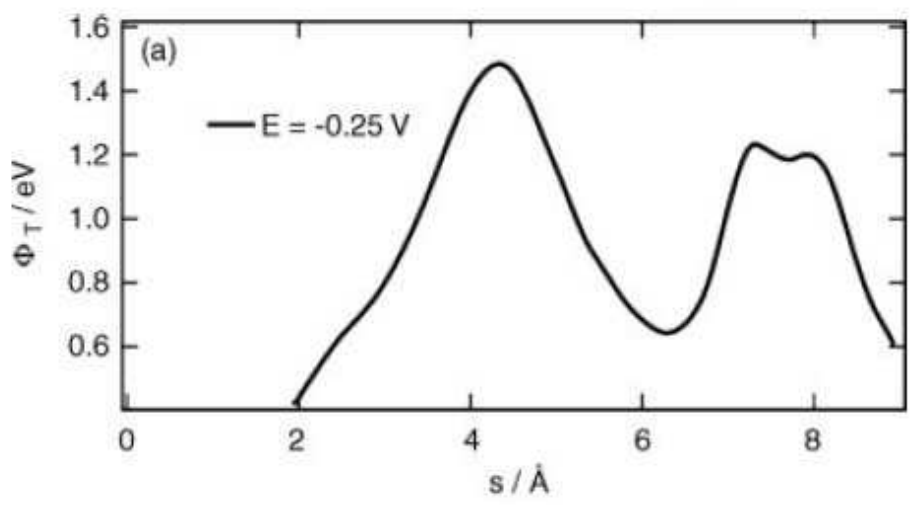



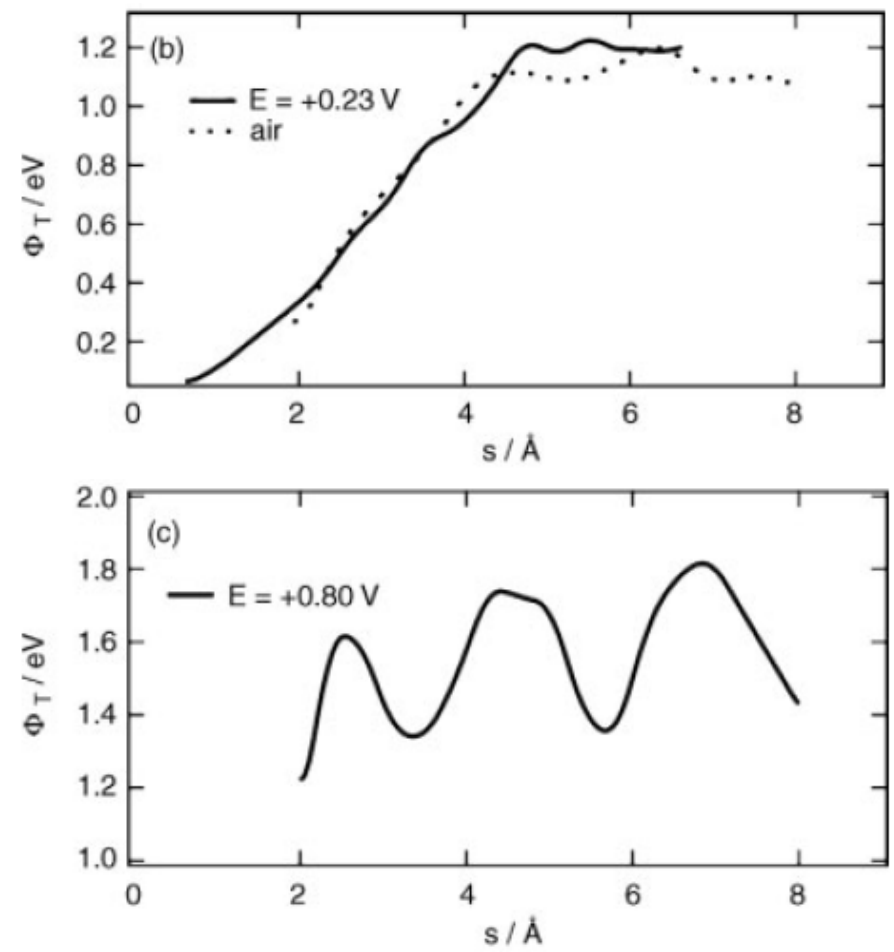

Fig.22: Tunneling barrier heights within the Helmholtz double layer at a $\mathrm{Au}(111)$ electrode; potentials as indicated (see text).

One observes that at pzc, the barrier height shows a linear behavior with distance as expected from the classical approach also seen in ambient air as indicated in Fig.22b. For cathodic polarization, pronounced variations of the barrier height that are in the order of $1 \mathrm{eV}$ are seen (Fig.22a). Although the pattern of the barrier height is different and less strongly modulated, the result in the anodic potential region also shows pronounced non-linear behavior. Here, three distinct maxima of $\Phi_{\mathrm{T}}$ are seen and the energy modulation is about $0.6 \mathrm{eV}$. These data can be related to the positions of the counter ions inside the double layer [45] and show that at least for non-adiabatic electron transfer where the tunneling probability is $<1$, tunneling trajectories can exist where the close approach of the nuclei is not necessary.

\section{Synopsis}

This overview of the experiments and interpretations of the work of D. M. Kolb on metal electrodes in conjunction with experiments on semiconductors that have been differently doped shows (i) electrochemical Stark effects where the energy of electronic states is altered by the Helmholtz layer potential and (ii) that the inner structure of the Helmholtz layer, even in the absence of specific adsorption, can play a crucial role in charge transfer processes due to pronounced variations in the barrier height for electron tunneling.

\section{Acknowledgements}

Support by the DFG, project LE 1192-4 and by the Joint Center for Artificial Photosynthesis is gratefully acknowledged. Dedicated to the memory of Dieter M. Kolb

\section{References}

1. O. Stern, Z.Electrochem. 30 (1924) 508

2. H. v. Helmholtz, Pogg.Ann. LXXXIX (1853) 211 
3. E.J.W. Verwey and J.T.G. Overbeek: Theory of the Stability of Lyophobic Colloids Elsevier, Amsterdam (1948).

4. A.V. Butler, Trans. Faraday Soc. 19 (1924) 729; ibid. 734

5. T. Erdey-Gruz, M. Volmer, Z. Phys. Chem. 150A (1930) 203

6. J. Tafel, Z. Phys. Chem. 50 (1905) 641

7. M. Stern, A.L. Geary, J. Electrochem. Soc. 104 (1957) 56

8. S. Trasatti, J. Electroanal. Chem. 39 (1977) 163

9. J. Greeley, J.K. Nørskov, L.A. Kibler, A.M. El-Aziz, D.M. Kolb, ChemPhysChem 7 (2006) 1032

10. J. Greeley, T.F. Jaramillo, J. Bonde, I. Chorkendorff, J.K. Nørskov, Nature Materials 5 (2006) 909

11. J. Newman, P.G. Hoertz, C.A. Bonino, J.A. Trainham, J. Electrochem. Soc. 159 (2012) 1722

12. Y. Tachibana, L. Vayssieres, J.R. Durant, Nature Photonics 6 (2012) 511

13. D.M.Kolb, W.Boeck, K.M.Ho, S.H.Liu, Phys. Rev. Lett. 47 (1981) 1921

14. W.Boeck, D.M.Kolb, Surf. Sci. 118 (1982) 613

15. K.-M.Ho C.-L.Fu, S.H.Liu, D.M.Kolb , G.Piazza, J. Electroanal. Chem. 150 (1983) 235

16. D.M.Kolb, F.C.Simeone, Electrochim. Acta 50 (2005) 2989

17. F.C.Simeone, D.M.Kolb, S.Venkatachalam, T. Jacob, Angew. Chem. Int. Ed. 46 (2007) 8903

18. H.Jungblut, J.Jakubowicz, S.Schweizer, H.J.Lewerenz, J. Electroanal. Chem. 527 (2002) 41

19. L.H. Thomas, Proc. Camb. Phil. Soc. 23 (1927) 542 ; E. Fermi, Rend. Accad. Naz. 6 (1927) 602

20. J.-N. Chazalviel: Coulomb Screening by Mobile Charges - Applications to Material Science, Chemistry and Biology, Birkhäuser, Boston (1999)

21. J.J. Benedetto, G. Zimmermann: J. Fourier Anal. Appl. 3 (1997) 505

22. J.N. Israelachvili: Intermolecular and Surface Forces, Academic Press $3^{\text {rd }}$ edition, (2011)

23. N.W. Ashroft, N.D. Mermin: Solid State Physics, HRW Internat. Editions (1976)

24. M.G. Evans, M. Polanyi, Trans. Faraday Soc. 31 (1935) 875

25. R.F. Mann, J.C. Amphlett, B.A. Peppley, C.P. Thurgood, J. Power Sources 2 (2006) 775

26. H. Wendt, V. Pizak, Electrochim. Acta 28 (1983) 27

27. P. Drude: Zur Elektronentheorie der Metalle. In: Ostwalds Klassiker der exaktenWissenschaften. Band 298, Deutsch Verlag, Frankfurt am (Main 2006)

28. J.Feinleib, Phys. Rev. Lett. 16 (1966) 1200

29. D.E.Aspnes, Surf. Sci. 37 (1973) 418

30. M.Cardona, K.Shaklee, F.H.Pollak, Phys. Rev. 154 (1967) 696

31. B.O.Seraphin, N.Bottka, Phys. Rev. 145 (1966) 628

32. D.M.Kolb, J.D.E.McIntyre, Surf. Sci. 28 (1971) 321

33. R. Koetz, H.J. Lewerenz, Surf. Sci. 97 (1980) 319

34. R. Koetz, H.J. Lewerenz, Surf. Sci. 78 (1978) L 233

35. H.J. Lewerenz, chapter 2 in: Advances in Electrochemical Science and Engineering, Vol. 12, pp.61-181, (eds. R. Alkire, D.M. Kolb, P.N. Ross), Wiley-VCH (2010)

36. R. Memming, G. Schwandt, Surf. Sci. 4 (1966) 109

37. H.J. Lewerenz, T. Bitzer, M. Gruyters, K. Jacobi, J. Electrochem. Soc. 140 (1993) L44

38. H. J. Lewerenz, M. Aggour, C. Murrell, M. Kanis, H. Jungblut, J. Jakubowicz, P. A. Cox, S. A. Campbell, P. Hoffmann, D. Schmeißer, J. Electrochem. Soc. 150 (2003) E 185 
39. J. Grzanna, H, Jungblut, H.J. Lewerenz, J. Electroanal. Chem. 486 (2000) 181 40. J. Grzanna, H. Jungblut, H.J. Lewerenz, J. Electroanal. Chem. 486 (2000) 190 41. J. Grzanna, T. Notz, H.J. Lewerenz, Phys. Stat. Sol. (c) 6 (2009) 1639 42. A. Natarajan, G. Oskam, P. Searson, J. Phys. Chem. B 102 (1998) 7793

43. H.J. Lewerenz, J. Stumper, L.M. Peter, Phys. Rev. Lett. 61 (1988) 1989

44. D.M.Kolb, F.C.Simeone, Electrochim. Acta 50 (2005) 2989

45. F.C.Simeone, D.M.Kolb, S.Venkatachalam, T. Jacob, Angew. Chem. Int. Ed. 46 (2007) 8903 